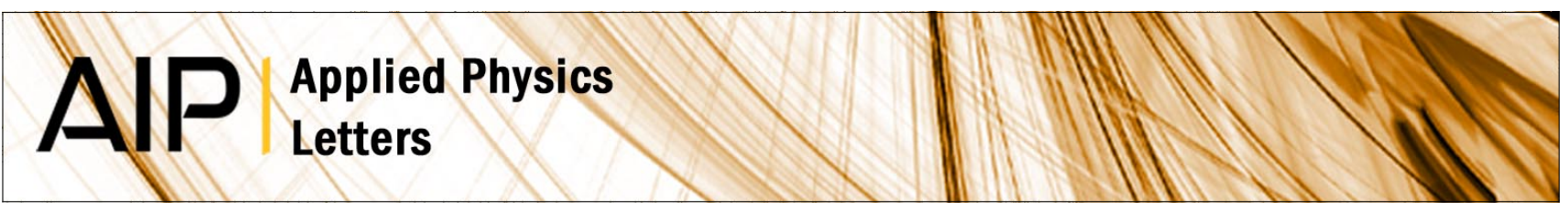

\title{
Light propagation in single mode polymer nanotubes integrated on photonic circuits
}

Nolwenn Huby, Jean Luc Duvail, Daphné Duval, David Pluchon, and Bruno Bêche

Citation: Appl. Phys. Lett. 99, 113302 (2011); doi: 10.1063/1.3637043

View online: http://dx.doi.org/10.1063/1.3637043

View Table of Contents: http://apl.aip.org/resource/1/APPLAB/v99/i11

Published by the American Institute of Physics.

\section{Related Articles}

Density and birefringence of a highly stable $\alpha, \alpha, \beta$-trisnaphthylbenzene glass

J. Chem. Phys. 136, 204501 (2012)

Molecular orientation and anisotropic carrier mobility in poorly soluble polythiophene thin films APL: Org. Electron. Photonics 5, 112 (2012)

Molecular orientation and anisotropic carrier mobility in poorly soluble polythiophene thin films Appl. Phys. Lett. 100, 203305 (2012)

Interplay between intrachain and interchain interactions in semiconducting polymer assemblies: The HJaggregate model

J. Chem. Phys. 136, 184901 (2012)

Excitation dynamics of a low bandgap silicon-bridged dithiophene copolymer and its composites with fullerenes APL: Org. Electron. Photonics 5, 90 (2012)

\section{Additional information on Appl. Phys. Lett.}

Journal Homepage: http://apl.aip.org/

Journal Information: http://apl.aip.org/about/about_the_journal

Top downloads: http://apl.aip.org/features/most_downloaded

Information for Authors: http://apl.aip.org/authors

\section{ADVERTISEMENT}

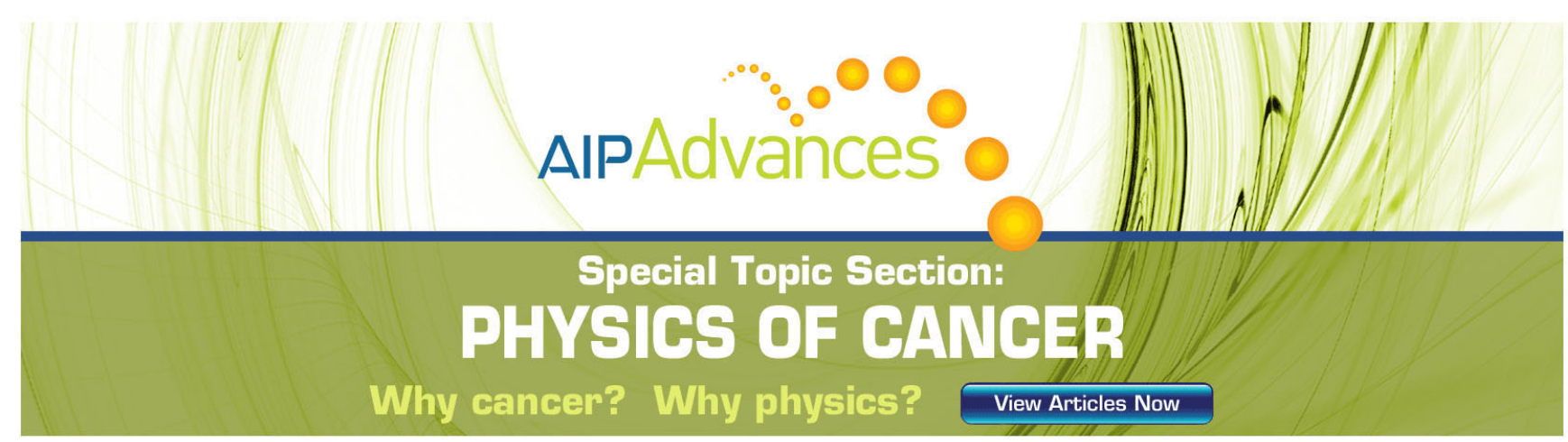




\title{
Light propagation in single mode polymer nanotubes integrated on photonic circuits
}

\author{
Nolwenn Huby, ${ }^{1, a)}$ Jean Luc Duvail, ${ }^{2}$ Daphné Duval, ${ }^{3}$ David Pluchon, ${ }^{1}$ and Bruno Bêche ${ }^{1}$ \\ ${ }^{1}$ Institut de Physique de Rennes, UMR CNRS 6251, Université de Rennes 1, 263 avenue Général Leclerc, \\ Rennes 35042, France \\ ${ }^{2}$ Institut des Matériaux, UMR CNRS 6502, Université de Nantes, 2 rue de la Houssinière B.P.32229, \\ 44322 Nantes cedex 3, France \\ ${ }^{3}$ Nanobiosensors and Bioanalytical Applications Group, CIN2 (CSIC-ICN) and CIBER-BBN, Campus UAB, \\ 08193, Bellaterra, Barcelona, Spain
}

(Received 15 July 2011; accepted 21 August 2011; published online 13 September 2011)

\begin{abstract}
We report the theoretical and experimental study of photonic propagation in organic dielectric nanotubes elaborated by a wetting template method and showing off an aspect ratio as high as 200 . Single mode behaviour is theoretically demonstrated without any cut-off conditions. Efficient evanescent coupling between polymer microstructures and nanotubes dispersed on a photonic chip as well as the high confinement and propagation in a single nanotube have been demonstrated. These results show the potential of well-defined one-dimensional nanostructures as building blocks for integrated organic photonic devices. Applications such as sensing and high speed communication are envisaged. (C) 2011 American Institute of Physics. [doi:10.1063/1.3637043]
\end{abstract}

Integrated photonic circuits offer great opportunities for high speed data transport and highly sensitive sensors. Related improvements rely on the comprehension of optical properties at the nanoscale and on the design of densely packed photonic devices. In this context, high aspect ratio nanostructures are mostly interesting. In particular, cylindrical nanostructures (nanowires and nanotubes NTs) always support at least one optical mode whatever their diameter. Moreover, they present a high evanescent field which can be exploited for highly sensitive sensors ${ }^{1}$ and for efficient coupling with photonic structures. ${ }^{2}$ Regarding the strategies for integrated photonics found in the literature, the ones based on polymeric materials are of great interest. Indeed, they present a large range of accessible optical properties by modification of their chemical structure. Moreover, liquid-phase processability and flexible support allow a large scale fabrication. Thereby, polymer-based photonic devices have already been reported such as organic light-emitting diodes, ${ }^{3}$ photodiode, ${ }^{4}$ solar cell, ${ }^{5}$ coupling splitters, ${ }^{6}$ or non-linear waveguides. ${ }^{7}$ Nevertheless, despite the increasing number of reports in nanophotonics involving 1D-nanostructures, dielectric organic nanotubes present a lack of investigations. Only a few in-solution fabrication methods are appropriate for tubular nanostructure elaboration. Among them, the wetting template method ${ }^{8}$ appears quite unique to precisely control the diameters of a large number of nanostructures processed in parallel in a non-consuming time, as evidenced recently for insulating ${ }^{9}$ and semi-conducting polymers. ${ }^{10,11}$

In this letter, we show photonic waveguiding behaviour in single organic SU8 (epoxy based negative photoresist) nanotube by a theoretical approach supplemented by an experimental investigation. This SU8 material has been already used at a micro-scale as light guiding structures, ${ }^{12}$ photonic crystal, ${ }^{13}$ and $\mathrm{MEMS}^{14}$ thus demonstrating it is an appropri-

\footnotetext{
${ }^{\text {a) }}$ Author to whom correspondence should be addressed. Electronic mail: nolwenn.huby@univ-rennes1.fr.
}

ate candidate for integrated optics. Its experimental investigation at the nanoscale is here performed by integrating SU8 nanotubes on a photonic chip also constituted of SU8 waveguiding structures.

The theoretical approach has been performed by applying to dielectric organic nanotubes an original analytical formalism recently developed. ${ }^{15}$ The modal cut-off thicknesses of the NTs are obtained by resolving numerically the eigenvalue equations. These cut-off values (outer $\phi_{\text {out }}$ and inner $\phi_{\text {in }}$ diameters) govern the presence or not of a mode in the structure. Figure 1 shows the cut-off values for the transverse electric $\mathrm{TE}_{01}$ and transverse magnetic $\mathrm{TM}_{01}$ modes. The normalized inner diameter $\phi_{i n} / \lambda$ is plotted as a function of the normalized outer diameter $\phi_{\text {out }} / \lambda$ resulting in a map of single mode and multimode areas, $\lambda$ being the wavelength. The geometrical limit depicts the forbidden zone where $\phi_{\text {in }}$ can not exceed $\phi_{\text {out }}$. The main result is that for $\phi_{\text {out }} / \lambda$ smaller than 0.64 , the structure is single hybrid $\left(\mathrm{HE}_{11}\right)$ whatever the inner diameter. For larger $\phi_{\text {out }} / \lambda$, additional modes are allowed

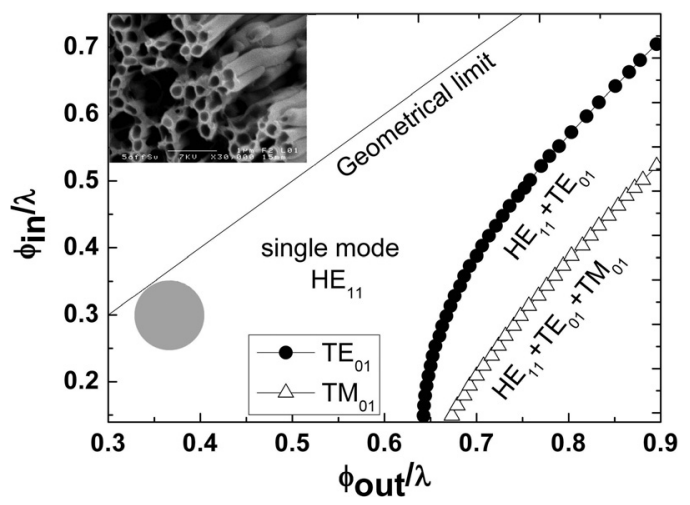

FIG. 1. Theoretical map of single mode and multimode behaviour in SU8 nanotubes as a function of inner $\phi_{\text {in }}$ and outer $\phi_{\text {out }}$ diameters normalized by the wavelength. The hatched grey zone highlights the area related to the nanotubes fabricated here $\left(\phi_{\text {out }}=255 \mathrm{~nm}\right.$ and $\left.\phi_{\text {in }}=215 \mathrm{~nm}\right)$. Insert: SEM image before the dispersion in single NTs. 
depending on the inner diameter. The grey hatched zone highlights the area related to NTs investigated in the following section. This map clearly proves that exclusively single mode propagation exists in these SU8 nanotubes.

The propagation of light into nanotubes with appropriate diameter has been experimentally investigated. SU8 nanotubes have been elaborated and integrated onto photonic micro-chips. Their fabrication is performed by a template strategy ${ }^{13}$ based on the impregnation of the $200 \mathrm{~nm}$-diameter pores of a commercially available $60-\mu \mathrm{m}$ thick alumina porous membrane (anodized aluminium oxide, AAO) with a drop of SU8 photoresist. After diffusion of the SU8 in pores, the whole sample was exposed to UV light $(\lambda=365 \mathrm{~nm}, 200$ $\mathrm{mJ} / \mathrm{cm}^{2}$ ) for polymerisation. To release the nanotubes from the alumina membrane, the sample was immersed in a selective $\mathrm{NaOH}$ solution $(1.5 \mathrm{M})$. Energy dispersive $\mathrm{x}$-ray spectroscopy analysis (not shown here) confirms the total removal of the AAO. The insert in Fig. 1 displays a scanning electron microscopy (SEM) image after the removal of the sample. From a statistical analysis of SEM images, the outer diameter $\phi_{\text {out }}$ of SU8 nanotubes is evaluated at $255 \pm 30 \mathrm{~nm}$ and the wall thickness at $40 \pm 10 \mathrm{~nm}$, which is consistent with the grey hatched zone of Fig. 1. Finally, the NTs are dispersed in single objects by sonication in isopropanol.

SU8 NTs have been integrated on a photonic integrated chip along organic SU8 microstructures. The fabrication process consists in a one-step UV-lithography process. The thickness of the microstructures (ridge waveguides of $6 \mu \mathrm{m}$ width and microdisk of $200 \mu \mathrm{m}$-radius connected to ridge waveguides) is $30 \mu \mathrm{m}$ and is determined by the viscosity of the SU8 (SU8-2025, $\eta=4500 \mathrm{~Pa}$ s) and by the patterning steps conditions. ${ }^{16}$ The final integrated photonic circuit is fully organic except the substrate working as the lower cladding layer $\left(n_{\mathrm{SiO}_{2}}=1.45\right)$. Schemes of the chip and of the micro-injection bench for optical characterization are presented in Fig. 2. The optical chip is tested on a set-up based on an end-fire method to couple light from the laser into the cleaved waveguide end facets. Visible light $(\lambda=670 \mathrm{~nm})$ and near infrared light $(\lambda=840 \mathrm{~nm})$ have been injected. Injection is controlled and optimized with a CCD camera at the exit of the chip, as shown on the CCD image where the cross-section of a waveguide during light propagation is presented. A micro-beam profiler (MBP, Newport) equipped by a CCD camera is placed on top of the bench allowing farfield intensity record.

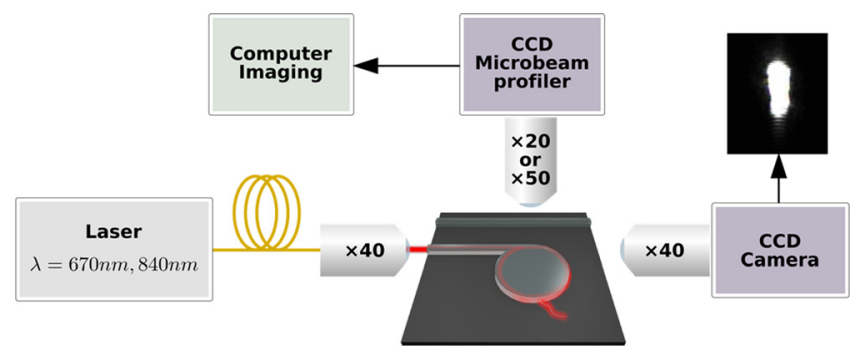

FIG. 2. (Color online) Sketch of the optical characterization set-up. Injection in integrated microstructures is optimized within cross-section imaging by a CCD camera (the CCD image shows the output of a waveguide of $30 \mu \mathrm{m}$ high and $6 \mu \mathrm{m}$ width). Top views of far field images are recorded with a CCD microbeam profiler.
Optical propagation in NTs is investigated through their excitation by evanescent coupling ${ }^{17}$ with a microdisk. In this purpose, light is coupled into a waveguide and propagates to the microdisk where whispering gallery modes (WGMs) are excited and circulate at the surface. This configuration is particularly appropriate since the evanescent tail of the guided light is highly dependent of the waveguide geometry. For a curved waveguide and as a consequence for a microdisk, the Gaussian beam axis shifts towards the outside of the bend ${ }^{18}$ and leads to an extended evanescent tail ${ }^{19}$ in the low refractive index medium (here, the air). Figure 3(a) shows a SEM image of a single NT located closed to a microdisk. Figure 3 (b) is the corresponding far-field intensity CCD image under infrared light injection. This close-up view, obtained with a 50x objective, demonstrates that light is confined both at the edges of the microdisk highlighting the WGM and along the NT. Figure 3(c) is an intensity profile plotted along the dotted white line visible in Fig. 3(b). The hatched rectangle has been added to depict the dimension of the nanotube. This profile shows the light confinement centered along the NT demonstrating the efficient optical evanescent coupling and the light propagation in these sub-wavelength nanostructures. It also highlights the high evanescent tail. Indeed, the fit of the profile with a Gaussian function leads to a full half width maximum of $739 \mathrm{~nm}$. The diameter of the NT of interest being $300 \mathrm{~nm}$ that leads to an extension of the field slightly higher than $200 \mathrm{~nm}$ outwards the NT.

The scattering losses have been quantitatively estimated from the exponential attenuation coefficient $\alpha$ of the BeerLambert law $I(x)=I_{0} \mathrm{e}^{-\alpha \mathrm{x}}$, where $I$ is the recorded light intensity along the propagation length $x$ (originating at the extremity of the NT close from the disk) and $I_{0}$ is the initial light intensity. Figure 3(d) shows the linear dependence $\ln$ $I=\mathrm{f}(x)$ along the NT during infrared light injection. The linear fit of the experimental data in Fig. 3(d) leads to losses of $210 \mathrm{~dB} / \mathrm{mm}$ for infrared light. The same study has been performed under red light injection and gives $129 \mathrm{~dB} / \mathrm{mm}$. This
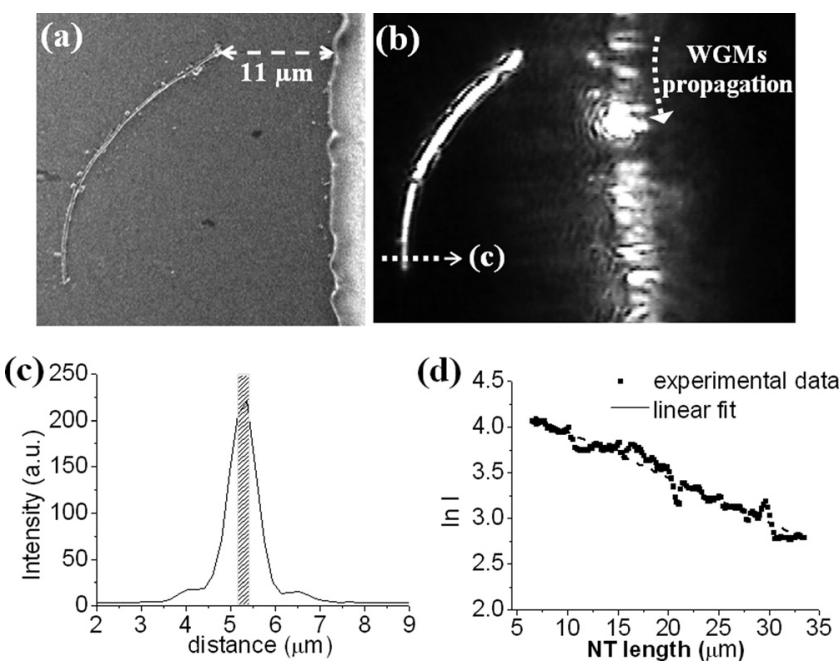

FIG. 3. (a) SEM image of a nanotube close to the edge of a disk-reservoir. (b) MBP image of the optical field during light injection. (c) Light intensity $I$ profile along the dotted line shown in (b). The hatched rectangle represents the NT diameter. (d) Experimental plot of $(\ln I)$ as a function of the distance along the NT. The slope of the linear fit leads to scattering losses estimation. 
wavelength dependence is consistent with previous studies evidenced in SU8 structures at a microscale. For magnitude losses comparison, SU8 microstructures exhibit few tenths of $\mathrm{dB} / \mathrm{mm}$ (Ref. 20) for red light. Higher losses in subwavelength waveguiding structures are expected due to the significant evanescent field. It can be noted that plasmon waveguides $^{21}$ present losses of few thousands $\mathrm{dB} / \mathrm{mm}$ and O'Carroll et al. reported losses of $480 \mathrm{~dB} / \mathrm{mm}$ in an active polymer nanowire waveguide. $^{22}$

In conclusion, we have demonstrated the waveguiding properties of dielectric organic nanotubes. The theoretical study predicts a $\mathrm{HE}_{11}$ monomode behaviour without frequency cut-off. Integration of SU8 NTs on an organic photonic chip constituted of appropriate microstructures for efficient evanescent coupling confirms their propagation capacity with acceptable losses compared to sub-wavelength nanostructures. The features of these SU8 nanotubes make them candidates for optical interconnections in highly packed and low-cost integrated optical circuits.

The authors thank A. Carré for technical support as well as J. Le Lannic for SEM images. These works were supported by Region Bretagne and Pays de la Loire, ANR-08JCJC-0136-01 programs and C'Nano Nord-Ouest network.

${ }^{1}$ K. Schmitt, K. Oehse, G. Sulz, and C. Hoffmann, Sensors 8, 711 (2008).

${ }^{2}$ Y. Li and L. Tong, Opt. Lett. 33, 303 (2008).
${ }^{3}$ Y. Huang, X. Duan, and C. M. Lieber, Small 1, 142 (2005).

${ }^{4}$ W. T. Hammond and J. Xue, Appl. Phys. Lett. 97, 073302 (2010).

${ }^{5}$ Z. Hu, B. Muls, L. Gence, D. A. Serban, J. Hofkens, S. Melinte, B. Nysten, S. Demoustier-Champagne, and A. M. Jonas, Nano Lett.7, 3639 (2007).

${ }^{6}$ A. Krishnan, C. J. Regan, L. Grave de Peralta, and A. A. Bernussi, Appl. Phys. Lett. 97, 231110 (2010).

${ }^{7}$ J. J. Ju, S. K. Park, S. Park, J. Kim, M.-S. Kim, M.-H. Lee, and J. Y. Do, Appl. Phys. Lett. 88, 241106 (2006).

${ }^{8}$ M. Steinhart, J. H. Wendorff, A. Greiner, R. B. Wehrspohn, K. Nielsch, J. Schilling, J. Choi, and U. Gösele, Science 296, 1997 (2002).

${ }^{9}$ M. Steinhart, J. H. Wendorff, and R. B. Wehrspohn, Chem. Phys. Chem. 4, 1171 (2003).

${ }^{10}$ F. Massuyeau, J. L. Duvail, J. M. Lorcy, S. Lefrant, J. Wery, and E. Faulques, Nanotechnology 20, 155701 (2009).

${ }^{11}$ D. H. Park, B. H. Kim, M. G. Jang, K. Y. Bae, and J. Joo, Appl. Phys. Lett. 86, 113116 (2005).

${ }^{12}$ J. Scheuer and A. Yariv, J. Eur. Opt. Soc. 1, 06007 (2006).

${ }^{13}$ M. De Vittorio, M. T. Todaro, T. Stomeo, R. Cingolani, R. D. Cojoc, and E. Di Fabrizio, Microelectron. Eng. 73, 388 (2004).

${ }^{14}$ T. Kondo, S. Juodkazis, and H. Misawa, Appl. Phys. A 81, 1583 (2005).

${ }^{15}$ D. Duval and B. Bêche, J. Opt. 12, 075501 (2010).

${ }^{16}$ B. Bêche, N. Pelletier, E. Gaviot, R. Hierle, A. Goullet, J. P. Landesman, and J. Zyss, Microelectron. J. 37, 421 (2006).

${ }^{17}$ D. Mulin, M. Spajer, D. Courjon, F. Carcenac, and Y. Chen, J. Appl. Phys. 87, 534 (2000).

${ }^{18}$ E. G. Neumann, IEE Proc. 129, 278 (1982).

${ }^{19} \mathrm{H}$. Nishihara, M. Haruna, and T. Suhara, in Optical Integrated Circuits (McGraw-Hill, New York, 1989), p. 43.

${ }^{20}$ M. Nordström, D. A. Zauner, A. Boisen, and J. Hübner, J. Lightwave Technol. 25, 1284 (2007).

${ }^{21}$ M. Hochberg, T. Baehr-Jones, C. Walker, and A. Scherer, Opt. Express 12, 5481 (2004).

${ }^{22}$ D. O'Carroll, I. Lieberwirth, and G. Redmond, Small 3, 1178 (2007). 